\title{
Preferential affinity of calcium ions to charged phosphatidic-acid surface from a mixed calcium/barium solution: X-ray reflectivity and fluorescence studies
}

\author{
Wei Bu, Kevin Flores, Jacob Pleasants, and David Vaknin* \\ Ames Laboratory, and Department of Physics and Astronomy, Iowa State University, Ames, Iowa 50011, USA
}

(Dated: October 29, 2018)

\begin{abstract}
X-ray reflectivity and fluorescence near total reflection experiments were performed to examine the affinities of divalent ions $\left(\mathrm{Ca}^{2+}\right.$ and $\left.\mathrm{Ba}^{2+}\right)$ from aqueous solution to a charged phosphatidicacid (PA) surface. A phospholipid (1,2-Dimyristoyl-sn-Glycero-3-Phosphate, DMPA), spread as a monolayer at the air/water interface, was used to form and control the charge density at the interface. We find that for solutions of the pure salts (i.e., $\mathrm{CaCl}_{2}$ and $\mathrm{BaCl}_{2}$ ), the number of bound ions per DMPA at the interface is saturated at concentrations that exceed $10^{-3} \mathrm{M}$. For a 1:1 $\mathrm{Ca}^{2+} / \mathrm{Ba}^{2+}$ mixed solutions, we find that the bound $\mathrm{Ca}^{2+} / \mathrm{Ba}^{2+}$ ratio at the interface is $4: 1$. If the only property determining charge accumulation near PA were the ionic charges, the concentration of mixed $\mathrm{Ca}^{2+} / \mathrm{Ba}^{2+}$ at the interface would equal that of the bulk. Our results show a clear specific affinity of PA for Ca compared to Ba. We provide some discussion on this issues as well as some implications for biological systems. Although our results indicate an excess of counterion charge with respect to the surface charge, that is, charge inversion, the analysis of both reflectivity and fluorescence do not reveal excess of co-ions (namely, $\mathrm{Cl}^{-}$or $\mathrm{I}^{-}$).
\end{abstract}

PACS numbers:

\section{INTRODUCTION}

Varying affinities among same-charge ions to counterions are expected and are commonly accounted for in chemistry. Although they are also common in biological processes, they are less pronounced because the strong tendency of the aqueous environment in biological systems to accentuate the electrostatic interactions rather than the chemical ones. However, close to charged interfaces, in channel proteins, or at high salt concentrations, the electrostatic description fails at the relevant short distances and quantum effects set in. We have undertaken the present study to determine the preferential affinity of $\mathrm{Ca}^{2+}$ or $\mathrm{Ba}^{2+}$ to a highly charged interface formed by phosphatidic acid (PA) using X-ray reflectivity and spectroscopy techniques. While PA is a minority lipid in cell membranes, it participates in numerous biological processes that are commonly triggered by small changes in Ca concentration. $\frac{1,2,3.4}{4}$ Molecular dynamics simulations have predicted that PA has a particular affinity to divalent ions via its oxygen atoms that may lead to charge inversion (namely, the charge density of the bound counterion exceeds that required to neutralize the surface charge exerted by PA) $\stackrel{\underline{5}}{\underline{n}}$ These simulations also predict that the PA becomes doubly charged by proton release. Recent $\mathrm{X}$-ray experiments on PA monolayers have revealed that charge accumulation of divalent ions and trivalent ions $s^{6.7}$ near PA interfaces show charge inversion, i.e. accumulation of charges that exceed the nominal surface charge. It is therefore important for both physical chemistry and biology to understand how calcium and other divalent ions interact with PA.

\footnotetext{
*vaknin@ameslab.gov
}

X-ray scattering techniques to determine ion distributions and binding to Langmuir monolayers $6.7,8,9,10,11,12,13,14,15,16,17,18,19,20$ (LM) have been extensively used to address the long standing problem of the structure of the electric double layer $21,22,23,24$ These include X-ray reflectivity $\underline{10,12}$ and anomalous reflectivity,$\stackrel{6,7,16,17}{17}$ which provide the total number of ions and the spatial ionic distributions at the charged interface. The near total X-ray reflection fluorescence (NTRF) technique monitors specifically and quantitatively ions near the interface $\frac{8,9,12,15,19,20}{19}$ but does not yield spatial information on the counterion layer. The near resonance X-ray energy scans at fixed momentum transfers can probe specifically the interfacial ions and yield their distributions as well as the $f^{\prime}(E)$ and $f^{\prime \prime}(E)$ fine structures 18 which, like the extended X-ray absorption fine structure technique (EXAFS), gives information on the local environment of the probed ion.

While monovalent ion distributions do not show appreciable specificity, as evidenced by the fact that their behavior are well described by PB theory that accounts for proton transfer and release ${ }^{21,22,23}$ from the charged headgroup, $\stackrel{16,17,18,26}{\text { distributions for multivalent ions }}$ show a high degree of ionic specificity ${ }^{27}$ The theoretical understanding of such ionic specificity is not fully understood. From classical statistical mechanics, it can be shown that Bjerrum pairing can account for some specificity related to the different sizes of the ions $\frac{28,29}{}$, but the general trends observed in many experiments suggest a much stronger specificity, thus requiring more complex quantum chemistry calculations.

Recently Shapovalov and coworkers ${ }^{20}$ applied X-ray fluorescence studies below the critical angle for total reflection to behenylsulfate monolayers that are spread on a variety of mixed ionic solutions to determine preferential association of ions to the highly charged sulfate head 
group. In their study of a $1: 1 \mathrm{Ca}^{2+} / \mathrm{Ba}^{2+}$ mixed solution, they find that $\mathrm{Ba}^{2+}$ cations have roughly 10 -fold preference over $\mathrm{Ca}^{2+}$ to associate with the sulfate headgroup. Although these authors argue that this result cannot be explained by the small difference in the hydration-sphere radii, recent theoretical work suggests the opposite ${ }^{30}$.

\section{EXPERIMENTAL SETUP AND METHODS}

Ion bulk concentrations were prepared using solutions of calcium chloride and iodide $\left(\mathrm{CaCl}_{2}, \mathrm{CaI}_{2}\right)$ and barium chloride $\left(\mathrm{BaCl}_{2}\right)$ obtained from Sigma-Aldrich. Each solution was mixed into ultra-pure water (Millipore apparatus; resistivity $18.1 \mathrm{M} \Omega \mathrm{cm}$ ). The surface charge density was controlled by a monolayer of 1,2Dimyristoyl-sn-Glycero-3-Phosphate (Monosodium Salt, DMPA; MW $=614$, from Avanti Lipids, Inc.). Small amounts (mili-grams) of DMPA were mixed into a 3:1 chloroform/methanol solution and spread at the surface of the bulk concentrations in a temperature controlled Langmuir trough. The trough is placed in a sealed canister that is kept at a constant temperature $\left(22^{\circ} \mathrm{C}\right)$ and is continuously purged with water-saturated helium gas. 10-15 minutes were allotted for solvent evaporation after which the monolayer was compressed at a rate of 1 $\AA^{2}$ per molecule per minute to the desired pressure (the experiments reported here were conducted at a constant pressure $\pi \approx 25 \mathrm{mN} / \mathrm{m}$ ). During the monolayer compression the surface pressure was recorded by a micro-balance using a filter-paper Wilhelmy plate. $\underline{\underline{6}}$

$\mathrm{X}$-ray reflectivity and fluorescence studies of monolayers at gas/water interfaces were conducted on a home built liquid surface X-ray reflectometer, using an UltraX18 Rigaku X-ray source generator with a copper rotating anode operating at $\sim 15 \mathrm{~kW}$ power. Down stream from the beam source, the characteristic $\mathrm{Cu} \mathrm{K} \alpha(\lambda=1.54$ $\AA$ ) is selected and deflected onto the liquid surface at a desired angle by a Ge(111) crystal. Scattered photon intensities were normalized to an incident beam monitor placed in front of the sample. The X-ray diffractometer was used in two configurations: reflectivity and fluorescence.

Specular X-ray reflectivity experiments yield the electron density (ED) profile across the interface, and can be related to molecular arrangements in the film: $\underline{31,32,33}$ The reflectivity for a LM at $Q_{z}$ is calculated by

$$
R\left(Q_{z}\right)=R_{0}\left(Q_{z}\right) e^{-Q_{z}^{2} \sigma^{2}},
$$

where $R_{0}\left(Q_{z}\right)$ is the reflectivity due to an ED profile composed of step functions, calculated by the recursive dynamical method, $\stackrel{34}{\underline{4}}$ and $\sigma$ is an effective surface roughness, which has a weak $Q_{z}$ dependence according to capillary wave theory applied to X-ray reflectivity studies ${ }^{35,36}$ In our reflectivity measurements, we use variable slit conditions that roughly keep the incident beam footprint constant on the liquid surface and also adjust the detector slit accordingly. This increases the angular accep- tance of the detector with $Q_{z}$, which makes the dependence of $\sigma$ on $Q_{z}$ large enough to require a correction. To a first order approximation, we correct for that by assuming $\sigma^{2}=a+b Q_{z}$, where $a$ and $b$ are two fitting parameters. Other variable parameters used to construct the ED across the interface include the thicknesses of the slabs $\left(d_{i}\right)$ and their corresponding electron densities $\left(\rho_{i}\right)$ (for details on the definitions of parameters see Ref. 17). The minimum number of slabs is the one for which the addition of another slab does not improve the quality of the fit, i.e. $\chi^{2}$, significantly.

To collect the fluorescence signal from the monolayercounterion system, we use an energy dispersive detector (X-PIPS Detector; model SXP8, with a Multiport II multichannel analyzer both from Canberra Industries Inc.). The pencil-like tip of the detector is lowered to the surface in an aluminum well in front of a thin Kapton window located about $\sim 2 \mathrm{~cm}$ above the liquid surface for fluorescence measurements (the fluorescence-setup is under the same conditions as for reflectivity, namely, the canister holding the trough is sealed and is purged with water saturated helium). The major advantage of the fluorescence technique is that it can distinguish between different elements by their characteristic emission lines. In the present study due to the low-yielded signal, the fluorescence signal is distinguished and displayed over two regions corresponding to two regions of the incident beam angles: below the critical angle for total reflection $\left(Q_{c} \sim 0.022 \AA^{-1}\right.$ with little variation due to salt in the solutions), which is highly sensitive to elements at the surface, and above the critical angle, which is sensitive to elements in the bulk and surface of the solution. Below the critical angle, the evanescent X-ray waves penetrate to $60-80 \AA$ from the surface, which makes the bulk contribution to the fluorescence intensity almost negligible at concentrations smaller than $10^{-3} \mathrm{M}$.

\section{EXPERIMENTAL RESULTS}

\section{A. Isotherms}

Figure 1 1 shows surface pressure versus molecular area isotherms for three different salt solutions (as indicated) at concentrations of $10^{-3} \mathrm{M}$ with DMPA and pure water with DMPA. The isotherm of DMPA on pure water lacks any distinct phase transitions with a smooth increase with compression. For DMPA spread on salt solutions, two slopes associated with crystalline tilted and untilted phases of the hydrocarbon chains with respect to the surface normal can be clearly identified. As the monolayer is compressed, it reaches the untilted stage $(\pi \approx 13 \mathrm{mN} / \mathrm{m})$ at the sufficiently low molecular area of $\sim 40 \AA^{2}$ per molecule. The isotherms of DMPA on 


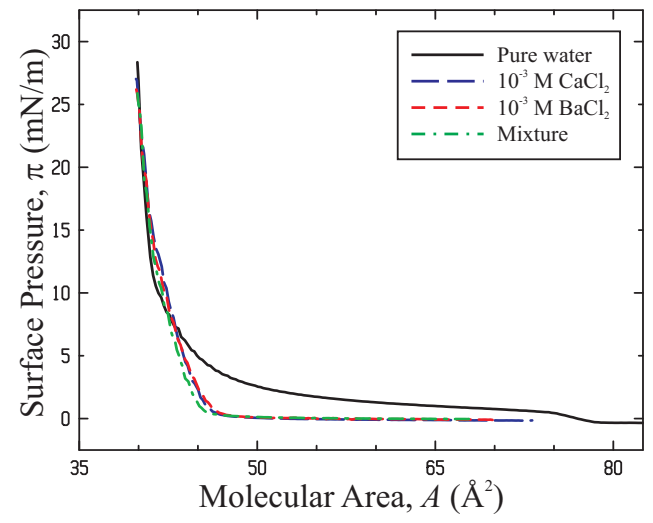

Figure 1: Surface-pressure versus molecular area $(\pi-A)$ isotherms of DMPA spread on water and dilute solutions, $10^{-3} \mathrm{M} \mathrm{CaCl}_{2}, \mathrm{BaCl}_{2}$, and a mixture solution $\left(5 \times 10^{-4} \mathrm{M}\right.$ $\left.\mathrm{CaCl}_{2}+5 \times 10^{-4} \mathrm{M} \mathrm{BaCl}_{2}\right)$. Although the isotherms on salts solution are different than that of water they are hardly distinguishable with no specific features that can be associated with the different ions.

the different salt solutions vary just slightly, exhibiting little or no specific features to the different ions in the solutions. All fluorescence and reflectivity measurements were conducted in the untilted crystalline stage $(\pi \sim 25$ $\mathrm{mN} / \mathrm{m})$.

\section{B. X-ray Reflectivity}

Normalized reflectivity data $\left(R / R_{F}\right.$, where $R_{F}$ is the calculated reflectivity of pure water with an ideally flat interface; $\sigma=0$ ) of DMPA monolayers spread on $10^{-3} \mathrm{M}$ solutions and on pure water are shown in Fig. 2. It is evident from Fig. 2 2 that the overall reflectivities for the samples prepared on salt solutions are significantly higher than that of the pure water data. This is a qualitative evidence that the ions $\left(\mathrm{Ba}^{2+}\right.$ and/or $\left.\mathrm{Ca}^{2+}\right)$ are accumulating at the surface. Also, there is a visible shift in the minima of the reflectivities to a smaller $Q_{z}$ for the solutions compared to water reflectivity, indicating a thicker layer due to the association of the ions at the headgroup region.

The normalized reflectivity data were fit (solid lines Fig. 2a and b) to a two slab model, one associated with the hydrocarbon chains of DMPA close to the vapor, and the other, contiguous to the bulk solution, associated with the phosphatidic-acid headgroup and bound ions and water molecules. The fitting parameters for the reflectivity data are listed in Table II. Using these parameters the electron density profiles for each solution and for pure water are obtained and plotted in Fig. 2r. It is interesting to note that the electron densities for divalent ion sub-phase are, within experimental uncertainties, identical. Qualitatively, this surprising result indicates there must be more calcium ions per DMPA
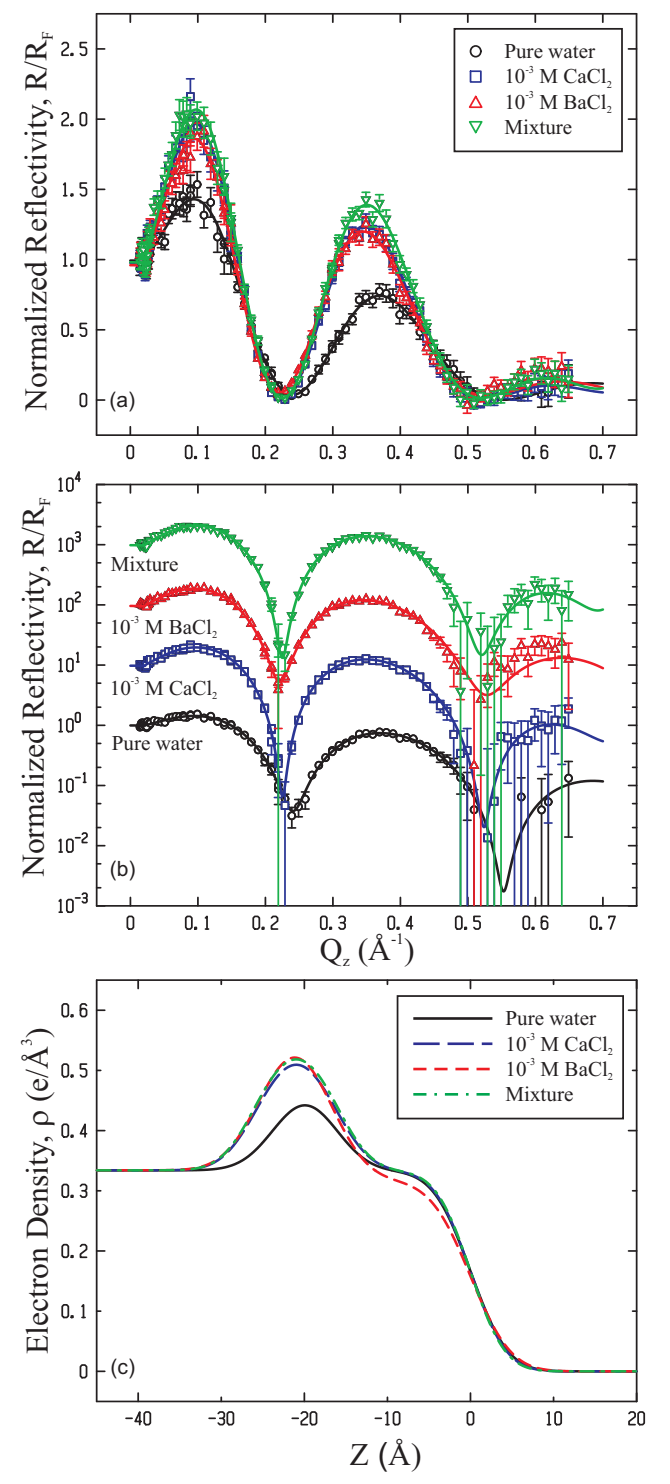

Figure 2: (a) Normalized reflectivity data $\left(R / R_{F}\right)$ versus momentum transfer $Q_{z}$ for each of the dilute solutions (as indicated) and on water with DMPA on a linear scale. (b) The same data on a logarithmic scale (curves and data are shifted by a decade each for clarity). (c) Electron density profiles used to calculate the best fits shown by solid lines in (a) and (b). The electron densities are generated with the parameters listed in Table 1

than barium ions as the two ions, $\mathrm{Ba}^{2+}$ and $\mathrm{Ca}^{2+}$, have very different numbers of electrons, 18 and 54, respectively. The phosphatidic-acid headgroup on pure water has a smaller thickness and smaller electron density than those obtained for the salt solutions, as expected since it does not have an electron-rich ion layer as with the salt solutions.

From the structural parameters in Table【, one can derive the number of ions per DMPA molecule at the surface by applying volume constraints and assuming the co-ions (e.g., $\mathrm{Cl}^{-}$) do not penetrate into the headgroup region (as 


\begin{tabular}{ccccc}
\hline sample & pure water & $10^{-3} \mathrm{M} \mathrm{CaCl}_{2}$ & $10^{-3} \mathrm{M} \mathrm{BaCl}_{2}$ & mixture \\
\hline$d_{\text {head }}$ & $5.4_{-0.9}^{+5.8}$ & $9.2_{-3.4}^{+1.6}$ & $7.1_{-1.5}^{+4.9}$ & $9.9_{-4.1}^{+1.1}$ \\
$\rho_{\text {head }}$ & $0.53_{-0.1}^{+0.22}$ & $0.55_{-0.05}^{+0.1}$ & $0.63_{-0.17}^{+0.06}$ & $0.55_{-0.03}^{+0.12}$ \\
$d_{\text {chain }}$ & $17.2_{-3.2}^{+0.5}$ & $16.3_{-0.8}^{+1.7}$ & $17.5_{-2.5}^{+1.0}$ & $16.0_{-0.6}^{+2.0}$ \\
$\rho_{\text {chain }}$ & $0.33_{-0.03}^{+0.01}$ & $0.33_{-0.02}^{+0.01}$ & $0.32_{-0.02}^{+0.01}$ & $0.33_{-0.02}^{+0.01}$ \\
$\sigma$ at $0.3 \AA^{-1}$ & $3.6_{-0.8}^{+0.4}$ & $3.4_{-0.4}^{+0.3}$ & $3.8_{-1.1}^{+0.1}$ & $3.3_{-0.3}^{+0.4}$ \\
$\#$ of ions & $\mathrm{N} / \mathrm{A}$ & $2.2_{-0.6}^{+0.1}$ & $0.9_{-0.6}^{+0.1}$ & $\mathrm{~N} / \mathrm{A}$ \\
$\#$ of water & $1.5_{-1.3}^{+9.4}$ & $6.8_{-5.6}^{+2.4}$ & $3.7_{-3.0}^{+6.4}$ & $\mathrm{~N} / \mathrm{A}$
\end{tabular}

Table I: Parameters used to fit the reflectivity data as well as the derived number of ions and water molecules at the interface. Uncertainties are obtained by fixing a parameter at values away from the optimum and readjusting all of the other parameters to a new minimum until $\chi^{2}$ increases by $25 \%$, and by imposing limiting values on some of the parameters to keep them within physical range; for instance, the electron density of the hydrocarbon chains was kept within $0.32 \pm 0.03$.

shown below). We use the following equations to solve for the number of water molecules $\left(n_{w}\right)$ and the number of ions $\left(n_{i}\right)$ per phosphatidic acid in the headgroup slab $\frac{37}{\underline{3}}$

$$
\begin{gathered}
A d_{h}=V_{h}+n_{w} V_{w}+n_{i} V_{i} \\
A d_{h} \rho_{h}=N_{h}+n_{w} N_{w}+n_{i} N_{i},
\end{gathered}
$$

where $A$ is the molecular area obtained from the isotherm and diffraction data,,$\frac{6,31.37}{} d_{h}$ and $\rho_{h}$ are the thickness and electron density of the head group, respectively, $N_{i}$ is the number of electrons per molecule (for water molecule $N_{w}=10$, and for $\mathrm{Ca}^{2+}$ and $\mathrm{Ba}^{2+} 18$ and 54 electrons, respectively). The molecular area can be confirmed self consistently by the electron density of the hydrocarbon chains $\rho_{\text {chain }}$ from the relation $A=N_{\text {chain }} /\left(d_{\text {chain }} \rho_{\text {chain }}\right)$. The volume of the phosphatidic headgroup, $V_{h}$, is obtained by the relations above, eqs. 2 and 3, using the the electron density profile parameters for DMPA spread on pure water. The precise position that parses the molecule into the hydrocarbon chain compartment and the headgroup region influences just slightly the derived parameters, and this change is accounted for in the uncertainties of the parameters given in Table I As seen in the table and as expected by the electron-density profiles, the number of $\mathrm{Ca}^{2+}$ and the number of $\mathrm{Ba}^{2+}$ ions per DMPA is not the same but rather seems to favor $\mathrm{Ca}^{2+}$ by $\mathrm{a} \sim 2: 1$ ratio for the unmixed salt solutions.

\section{Fluorescence}

Fluorescence scans were performed on all the solutions with and without DMPA, and the background data was obtained from a pure water sample without DMPA and subtracted from all data to remove electronic and stray signals from the trough. The data from the fluorescence
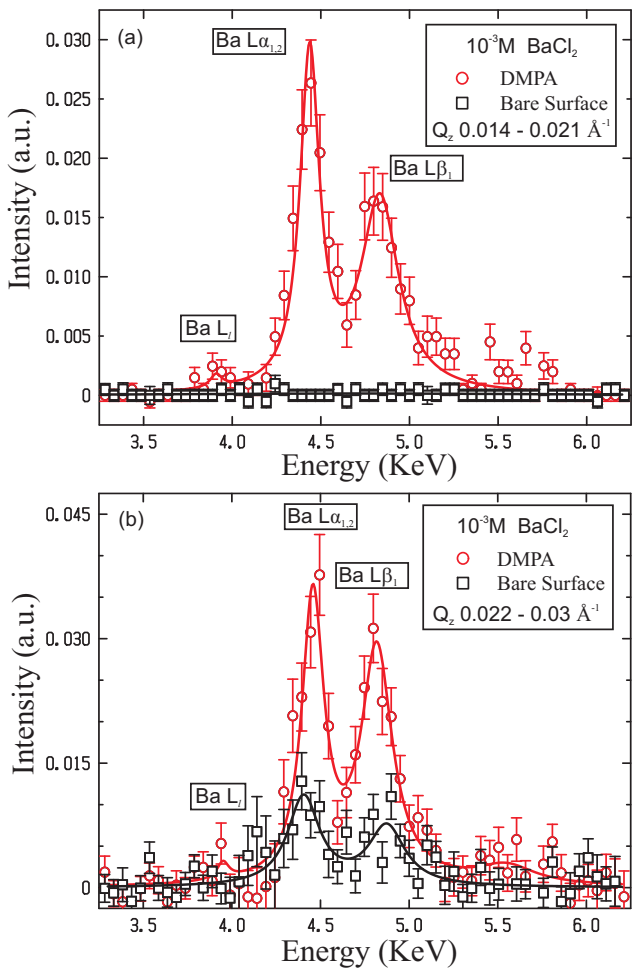

Figure 3: (a) Fluorescence signals below the critical angle for $10^{-3} \mathrm{M} \mathrm{BaCl}_{2}$ of the solution only and the same solution with the DMPA monolayer as indicated. (b) Fluorescence data above the critical angle from the same two samples. The signals above and below the critical angle were integrated over a $Q_{z}$ range as indicated in the figures.

were then integrated over $Q_{z}$ ranges to improve signal to noise ratio as described above. Figure 3 shows fluorescence data below (a) and above (b) the critical angle for $10^{-3} \mathrm{M} \mathrm{BaCl}_{2}$ with and without DMPA, respectively. The intensity was integrated over the range shown in the figures. As shown in Fig. 3(a) (below the critical angle), without a monolayer the evanescent X-ray beam does not penetrate far enough into the bulk to generate any visible fluorescence intensity. However, with the DMPA monolayer on, the $\mathrm{L} \alpha(4.46 \mathrm{keV})$ and $\mathrm{L} \beta_{1}(4.8 \mathrm{keV})$ emission lines for $\mathrm{Ba}^{2+}$ are clearly visible, which indicates that the concentration of ions at the interface is much larger compared to that of the bulk concentration. In Fig. 3(b), emission lines can be seen in both the solution without and with DMPA. However, the solution with the monolayer has an enhancement for each emission line as the ions at the surface contribute significantly to the overall intensity. The difference in the intensities of the two data sets in Fig. 3(b) quantifies the contribution of the ions at the surface. Spectra of $\mathrm{K} \alpha \approx 3.7 \mathrm{keV}$ and $\mathrm{K} \beta \approx 4.03$ $\mathrm{keV}$ emission lines for the $\mathrm{CaCl}_{2}$ solution (not shown) essentially show similar trends. Figure 4 shows that the emission lines below the critical angle (contributed primarily by surface) are practically identical at $10^{-3} \mathrm{M}$ and $10^{-2} \mathrm{M} \mathrm{BaCl}_{2}$, indicating that the monolayer is saturated with ions for concentrations stronger than $10^{-3} \mathrm{M}$. 


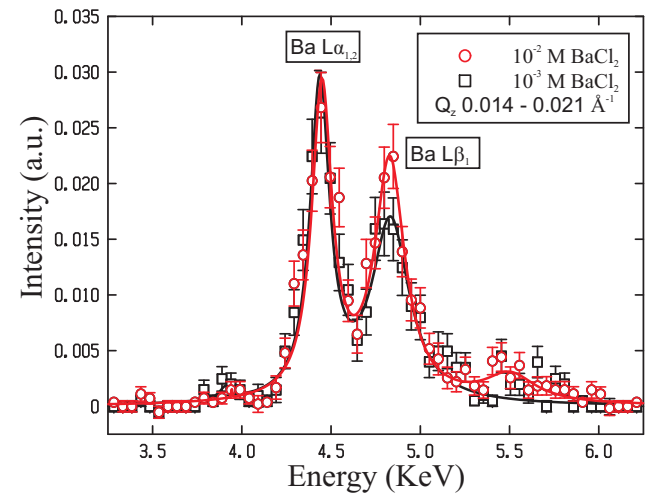

Figure 4: Integrated fluorescence signals below the critical angle for solutions of $10^{-2} \mathrm{M}$ and $10^{-3} \mathrm{M} \mathrm{BaCl}_{2}$ showing the binding of ions reaches saturation for concentrations $\lesssim 10^{-3}$ M.

$\mathrm{CaCl}_{2}$ and the mixture solutions display similar binding phenomena at these concentrations.

To get more quantitative results we define a "standard ratio" as the ratio of fluorescence signals for specific emission lines from two different elements in equal quantities in the bulk solution (e.g., $\mathrm{Ca}^{2+} / \mathrm{Ba}^{2+}$ ). From Fig. $5(\mathrm{a})$, pure solutions without DMPA, we establish a "standard ratio" (by comparing the peak intensities of $\mathrm{Ba}^{2+} \mathrm{L} \alpha$ and $\mathrm{Ca}^{2+} \mathrm{K} \alpha$ ) of the emission lines generated by equal concentrations of $\mathrm{Ba}^{2+}$ and $\mathrm{Ca}^{2+}$ ions at $\sim 6: 1$ in favor of $\mathrm{Ba}^{2+}$. Comparing the ratio between the same peak intensities provides the relative population of each ion at the interface. From Fig. 5 (b) we find the $\mathrm{Ba}^{2+} / \mathrm{Ca}^{2+}$ ratio of the pure solutions reduces to $\sim 3: 1$ and for the mixed solution the ratio is even lower $\sim 1.5: 1$. This shows that $\mathrm{Ca}^{2+}$ in unmixed solution populates the PA interface twice as much as the $\mathrm{Ba}^{2+}$ does, consistent with $\mathrm{X}$-ray reflectivity results. In the $\mathrm{Ba} / \mathrm{Ca} 1: 1$ mixed solutions calcium ions are more likely to associate with the $\mathrm{PA}$ at the interface by a ratio of $\sim 4: 1$ over $\mathrm{Ba}^{2+}$.

\section{DISCUSSION AND CONCLUSIONS}

The objective of this study has been to explore the binding specificity of divalent ions in solutions to a phosphatidic acid charged surface and in particular, to determine which of the two $\mathrm{Ba}^{2+}$ or $\mathrm{Ca}^{2+}$ is more favorable to binding to the DMPA monolayer. Our results show that, in a 1:1 mixed solution, $\mathrm{Ca}^{2+}$ is favored over $\mathrm{Ba}^{2+}$ by almost a $4: 1$ ratio. This is strikingly different from the results of Shapovalov and coworkers ${ }^{20}$ who found that $\mathrm{Ba}^{2+}$ outnumbers $\mathrm{Ca}^{2+}$ by a $10: 1$ in binding to a behenylsulfate monolayer. Clearly, the only possible origin of such a discrepancy is a strong specificity of ionic and
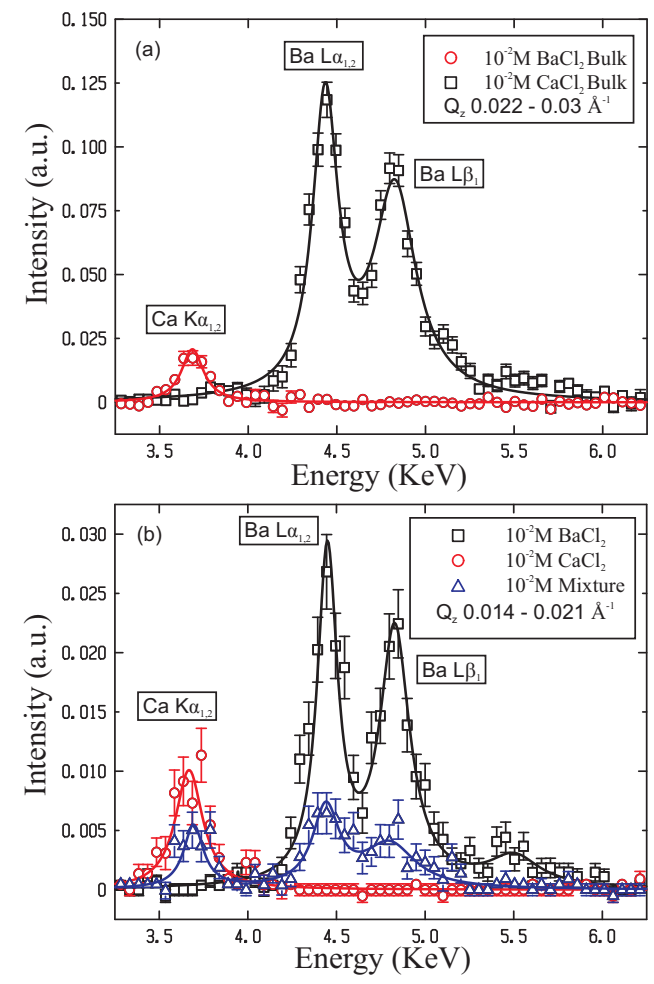

Figure 5: (a) Integrated fluorescence signals above the critical angle showing the relative intensities of $\mathrm{Ba}$ and $\mathrm{Ca}$ emission lines from the solutions only at $10^{-2} \mathrm{M}$ (no monolayer). This data is used to calibrate the relative intensities from the same number of $\mathrm{Ba}$ and $\mathrm{Ca}$ ions. (b) Integrated fluorescence signals below the critical angle for DMPA monolayers spread on $\mathrm{BaCl}_{2}, \mathrm{CaCl}_{2}$ and 1:1 mixture at a nominal $10^{-2} \mathrm{M}$. This indicates the amount of bound $\mathrm{Ba}^{2+}$ to $\mathrm{PA}$ is significantly smaller than that of $\mathrm{Ca}^{2+}$ for DMPA on the mixture.

headgroup interactions, and cannot be quantitatively accounted for by theories based on classical electrostatic interactions, even if the ionic size is properly accounted for. Furthermore, our finding that $\mathrm{Ca}^{2+}$ binds more strongly than $\mathrm{Ba}^{2+}$ can only be understood if the ion dehydrates upon binding. Quantitative theoretical account for these effects remains an outstanding theoretical problem.

Our results also imply charge inversion at the surface in particular, for the $\mathrm{CaCl}_{2}$ and $\mathrm{CaI}_{2}$ solutions, as shown below. This happens when the charge density of the counterions exceeds the surface charge density exerted by the phosphatidic monolayer. Our analysis shows there there is one $\mathrm{Ba}^{2+}$ per DMPA molecule, which is just enough to neutralize the surface charges (doubly charged phosphatidic acid), whereas there is more than one $\mathrm{Ca}^{2+}$ ion per DMPA molecule at the surface. This suggests charge inversion, from negatively charged to positively charged interface. In the fluorescence experiments above, we did not detect strong emission signals from $\mathrm{Cl}^{-}$to suggest that there exists an accumulation of the co-ion $\left(\mathrm{Cl}^{-}\right)$at the interface due to charge inversion. To further explore if the anions are rising to the charge-inverted surface, we performed another experiment using $10^{-2} \mathrm{M} \mathrm{CaI}_{2}$ solu- 

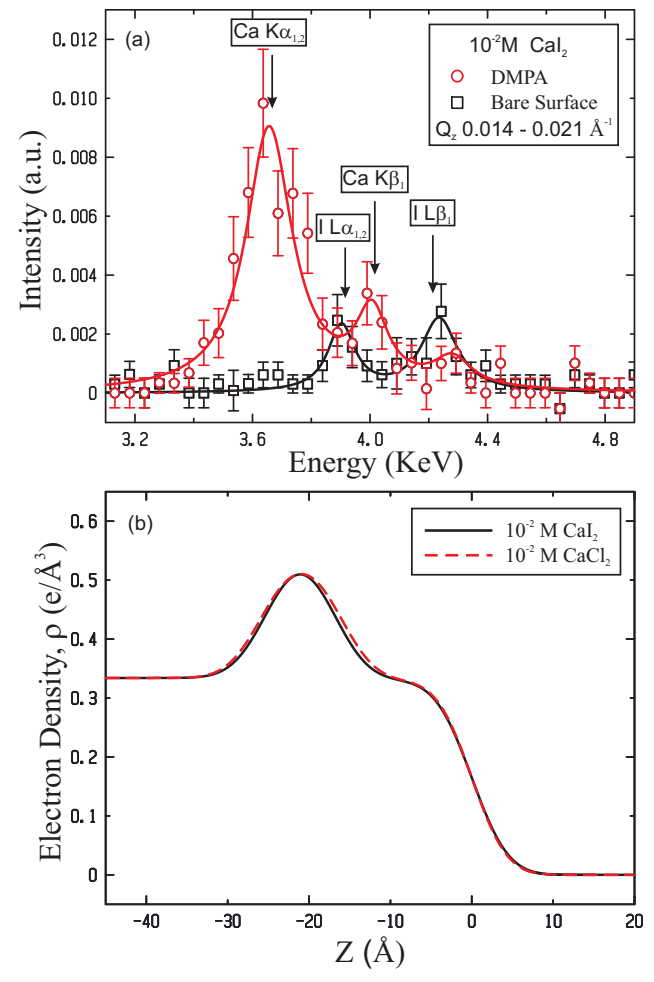

Figure 6: (a) Fluorescence signals from the surface of $\mathrm{CaI}_{2}$ $\left(10^{-2} \mathrm{M}\right)$ solution only, and the same solution with the DMPA monolayer on. At this relatively high concentration the signal from iodine is clearly seen before spreading the monolayer whereas the signal form calcium is very weak to detect. With the DMPA monolayer on, the Ca signal is enhanced whereas the signal from the iodine slightly weakens. This shows there is no enrichment of the iodine co-ion at the interface due to possible charge-inversion. (b) The electron density profiles for films of DMPA spread on $10^{-2} \mathrm{M} \mathrm{CaI}_{2}$ and $10^{-2} \mathrm{M} \mathrm{CaCl}_{2}$ solutions. This shows the amount of $\mathrm{I}^{-}$is negligible at the interface although the number of $\mathrm{Ca}^{2+}$ ions exceeds those necessary to neutralize the phosphatidic-acid. tion with DMPA as a monolayer to improve the fluorescent signal from the co-ion, in this case from $\mathrm{I}^{-}$. Figure 6)(a) shows spectra below the critical angle, indicating surface enrichment of $\mathrm{Ca}^{2+}$ but no significant difference in the emission lines of $\mathrm{I}^{-}$before and after the spreading of DMPA. We have also measured the reflectivity from the same DMPA monolayer on $\mathrm{CaI}_{2}$ solution to detect any changes in the electron density near the headgroup region compared to that of DMPA on $\mathrm{CaCl}_{2}$ solution, that may indicate the accumulation of $\mathrm{I}^{-}$at the interface. Figure 6(b) shows the ED profiles of the films on both solutions $\left(\mathrm{CaCl}_{2}\right.$ or $\left.\mathrm{CaI}_{2}\right)$ are practically the same. We therefore conclude that if there are co-ions (iodine or chlorine ions) at the surface due to charge inversion, their concentration is below 0.1 iodine ion per PA molecule according to our detection sensitivity, or that the $\mathrm{Ca}^{2+}$ ions migrate to the surface partially as $\mathrm{CaOH}^{+}$.

To summarize, in numerous physiological studies such as those of the 'Ca receptor' in preganglionic nerve terminals (interacting primarily with acetyl-choline) it has found that $\mathrm{Ba}^{2+}$ ions, do not appear to compete with $\mathrm{Ca}^{2+}$ for the receptor ${ }^{38}$. This is consistent with our studies that show stronger affinity of Ca over Ba to negatively charged PA interface. Systematic studies of model systems using spectroscopic X-ray techniques that are ion specific open the door to unraveling the mechanism of this and related physiological phenomena.

\section{Acknowledgments}

We wish to thank Alex Travesset for helpful discussions during the course of this work, and for his comments and suggestions on the manuscript. This manuscript has been authored, in whole or in part, under Contract No. DEAC02-07CH11358 with the U.S. Department of Energy.
1 Meijer, H. G. H.; Munnik, T. Annu. Rev. Plant Biol. 2003, 54, 265.

${ }^{2}$ Ishii, I.; Fukushima, X. Ye.; Chun, J. Annu. Rev. Biochem. 2004, 73, 321.

3 Wang, X.; Devaiah, S. P.; Zhang, W.; Welti, R. Prog. Lipid Res. 2006, 45, 250.

${ }^{4}$ Kooijman, E. E.; Tielman, D. P.; Testerink, C.; Munnik, T.; Rijkers, D. T. S.; Burger, K. N. J.; de Kruiiff, B. J. Biol. Chem. 2007, 282, 11356.

5 Faraudo, J,; Travesset, A. Biophys. J. 2007, 92, 2806; and Coll. Surf. A 2007, 300, 287.

6 Vaknin, D.; Kruger, P.; Losche, M. Phys. Rev. Lett. 2003, 90, 178102/1-4.

7 Pittler, J.; Bu, W.; Vaknin, D.; Travesset, A.; McGillivray, D. J.; Losche, M. Phys. Rev. Lett. 2006, 97, 046102/1-4.

8 Bloch, J. M.; Sansone, M.; Rondelez, F.; Peiffer, D. G.; Pincus, P.; Kim, M. W.; Eisenberger, P. M. Phys. Rev. Lett. 1985, 54, 1039.

9 Bloch, J.M.; Yun, W.B.; Yang, X.; Ramanathan, M.; Mon- tano, P. A.; Capasso, C. Phys. Rev. Lett. 1988, 61, 2941.

10 Kjaer, K.; Als-Nielsen, J.; Helm, C.; Tippman-Krayer, P.; Mohwald, H. J. Phys. Chem. 1989, 93, 3200.

11 Jun, J. W.; Bloch, J. M. J. Appl. Phys. 1990, 68, 1421.

12 Daillant, J.; Bosio, L.; Benattar, J. J.; Blot, C. Langmuir 1991, 7, 611.

13 Jacquemain, D.; Wolf, S. G.; Leveiller, F.; Deutsch, M.; Kjaer, K.; Als-Nielsen, J.; Lahav, M.; Leiserowitz, L. Angew. Chem. 1992, 31, 130.

14 Novikova, N. N.; Zheludeva, S. I.; Konovalova, O. V.; Kovalchuk, M. V.; Stepina, N. D.; Myagkov, I. V.; Godovsky, Y. K.; Makarova, N. N.; Tereschenko, E. Y; Yanusova, L. G. J. Appl. Crystallogr. 2003, 36, 727.

15 Zheludeva, S. I.; Novikova, N. N.; Konovalov, O. V.; Kovalchuk, M. V.; Stepina, N. D.; Tereschenko, E. Y. Mater. Sci. Eng. C 2003, 23, 567.

${ }^{16}$ Bu, W.; Vaknin, D.; Travesset, A. Phys. Rev. E 2005, 72, 060501(R)/1-4.

17 Bu, W.; Vaknin, D.; Travesset, A. Langmuir 2006, 22, 
5673.

18 Bu, W.; Ryan, J. P.; Vaknin, D. J. Synchrotron Rad. 2006, $13,459$.

19 Shapovalov, V. L.; Brezesinski, G. J. Phys. Chem. B 2006, 110, 10032.

${ }^{20}$ Shapovalov, V. L.; Ryskin, M. E.; Konovalov, O. V.; Hermelink, A.; Brezesinski, G. J. Phys. Chem. 2007, 111, 3927.

21 Ninham, B W.; Parsegian, V. A.; J. Theor. Biol. 1971, 31, 405.

${ }^{22}$ Bloch, J. M.; Yun, W. Phys. Rev. A 1990, 41, 844.

${ }^{23}$ Israelachvili, J. Intermolecular and surface forces, Academic Press, London, 2000.

24 Andelman, D. Handbook of Biological Physics, vol. I, R. Lipowsky.; E. Sackmann (Eds.), Electrostatic Properties of Membranes: The Poisson-Boltzmann Theory, p 603-641 Chapter 12 (Elsevier Science, Amsterdam, 1995).

${ }^{25}$ Luo, G. M.; Malkova, S.; Yoon, J.; Schultz, D. G.; Lin, B. H; Meron, M.; Benjamin, I.; Vanysek, P.; Schlossman, M. L. Science 2006, 311, 216.

${ }^{26}$ Giewekemeyer K.; Salditt, T. Euro. Phys. Lett. 2007, 79, 18003/1-18003/6.

27 Koelsch, P.; Viswanath, P.; Motschmann, H.; Shapovalov, V. L.; Brezesinski, G.; Möhwald, H.; Horinek, Do- minik; Netz, Roland R.; Giewekemeyer, K.; Alditt, T. S.; Schollmeyer, H.; von Klitzing, Regine.; Daillant, J.; Guenoun, P. Coll. Surf. A 2007, 303, 110.

28 Travesset, A.; Vaknin, D. Europhys. Lett. 2006, 74, 181.

29 Faraudo, J.; Travesset, A. J. Phys. Chem. C 2007, 111, 987.

30 Biesheuvel, P. M.; van Soestbergen, M. J. Coll. and Inter. Sci. 2007, 316, 490.

31 Vaknin, D.; Kjaer, K.; Als-Nielsen, J.; Losche, M. Biophys. J. 1991, 59, 1325.

32 Kjaer, K. Physica B 1994, 198, 100.

33 Vaknin, D. Characterization of Materials, Kaufmann, E. N., Ed,; Wiley: New York, 2003; Vol. 2, p 1027.

34 Parratt, L. G. Phys. Rev. 1954, 59, 359.

35 Braslau, A.; Pershan, P. S.; Swislow, G.; Ocko, B. M.; Als-Nielsen, J. Phys. Rev. A 1988, 38, 2457.

36 Ocko, B. M.; Wu, X. Z.; Sirota, E. B.; Sinha, S. K.; Deutsch, M. Phys. Rev. Lett. 1994, 72, 242.

37 Gregory, B. W.; Vaknin, D.; Gray, J. D.; Ocko, B. M.; Stroeve, P.; Cotton, T. M.; Struve, W. S. J. Phys. Chem. B 1997, 101, 2006.

38 E. M. McLachlan, E. B.; J. Physiol. 1977, 267, 497. 